\title{
O custo social e econômico dos acidentes de trânsito com pedestres e ciclistas: estudo de caso do estado de Santa Catarina, Brasil
} The social and economic cost of traffic accidents with pedestrians and cyclists:
case study of Santa Catarina state, Brazil

Vinicius Tischer[a] (1)

[a] Universidade do Vale do Itajaí (UNIVALI), Itajaí, SC, Brasil

Como citar: Tischer, V. (2019). O custo social e econômico dos acidentes de trânsito com pedestres e ciclistas: estudo de caso do estado de Santa Catarina, Brasil. urbe. Revista Brasileira de Gestão Urbana, 11, e20180029. https://doi.org/10.1590/2175-3369.011.001.A012

\section{Resumo}

Os custos sociais decorrentes de acidentes de trânsito são elevados e impactam diretamente na economia. Entre as principais vítimas desses acidentes estão ciclistas e pedestres, os usuários mais vulneráveis. Nesse sentido, o objetivo foi desenvolver uma metodologia para estimar os custos gerados pelos acidentes de trânsito envolvendo pedestres e ciclistas. 0 estudo teve como referência dados disponíveis no Sistema de Informações de Saúde, vinculado ao Ministério da Saúde, considerando vítimas fatais e não fatais de acidentes. Para a composição dos custos de vítimas não fatais, foram utilizadas duas abordagens: uma considerando apenas despesas médico-hospitalares e outra levando em conta também os custos indiretos. Para acidentes fatais, empregou-se o método do capital humano, utilizando rendimentos fornecidos pelo IBGE como base para os cálculos. Como resultado, obtiveram-se diferentes aproximações de custos para cada tipo de ocorrência (lesões e vítimas fatais), demonstrando o ônus resultante do elevado número de acidentes. Os resultados obtidos podem ser utilizados em subsídio ao planejamento estratégico urbano e às políticas de segurança nos deslocamentos.

Palavras-chave: Acidentes de trânsito. Custos e análise de custo. Pedestres. Ciclistas.

\section{Abstract}

The social costs resulting from traffic accidents are high and have a direct impact on the economy. Among the main victims of these accidents are cyclists and pedestrians, consisting of the most vulnerable users. Thus, the purpose of this paper is to develop a methodology to estimate the costs generated due to traffic accidents involving pedestrians and cyclists. This study had as reference data contained in the Health Information system which part is from National Ministry of Health, considering fatal and non-fatal accident victims. For the composition of the costs of non-fatal victims, two approaches were used, one considering only medical-hospital expenses and the other also considering indirect costs. For fatal accidents the Human Capital method was used, using income provided by the IBGE as a basis for calculations. As a result, different cost approaches were obtained for each type of occurrence (injuries and

VT é engenheiro, bacharel em Engenharia Ambiental e mestre em Ciências e Tecnologia Ambiental, e-mail: tischer@edu.univali.br 
fatalities), demonstrating the charge resulting from the high number of accidents. The results obtained can be used to subsidize the urban strategic planning and safety policies on commuting.

Keywords: Traffic accidents. Costs and cost analysis. Pedestrians. Cyclists.

\section{Introdução}

O modelo de transporte e de mobilidade urbana empregado atualmente no Brasil favorece a utilização de transporte motorizado individual. Segundo a Bazani (2016), no ano de 2014, 31\% das viagens foram realizadas por meio de transportes individuais, correspondendo à parte expressiva dos deslocamentos urbanos. No geral, as políticas e os investimentos públicos fomentam o uso de carros em detrimento do transporte público e dos modos ativos, como o uso de bicicletas (Newman \& Kenworthy, 2013; Tischer, 2017).

Esse modelo de desenvolvimento combina fatores que contribuem para uma redução da qualidade dos deslocamentos, como as baixas condições de segurança (alta velocidade em vias), a qualidade insatisfatória dos transportes públicos e a elevada frota de veículos particulares, e tem como um dos principais resultantes as elevadas taxas de acidentes de trânsito (Bacchieri \& Barros, 2011; Biffe et al., 2017). É notável destacar que a precariedade do sistema de transporte público, principalmente em países emergentes, potencializa os riscos de acidentes de trânsito, sendo verificado que, quanto mais viagens de transporte público, menor é a incidência de acidentes graves no trânsito (Litman, 2008, 2014).

Os custos sociais decorrentes de acidentes de trânsito são elevados para a sociedade. Em especial os envolvendo automóveis representam, em vários locais do mundo, a principal causa de morte não natural. Em âmbito mundial, cerca de 1,3 milhão de mortes acontecem anualmente devido a acidentes de trânsito, havendo um número maior ainda de internações, atendimentos em serviços de emergência e sequelas físicas e psicológicas (OECD, 2015).

Além desses números elevados de acidentes e das consequências psicológicas provocadas pela perda de vidas, os impactos econômicos afetam profundamente a economia nacional, tanto diretamente (pelos custos médico-hospitalares) como indiretamente (por exemplo, pelos custos de oportunidades perdidas) (Lima, 2003; IPEA, 2015; Garcia et al., 2013; Andrade et al., 2017).

O impacto econômico pode ser exemplificado pelos dados do boletim estatístico da Seguradora Líder DPVAT (Seguradora Lider, 2016), os quais demostram que, em 2016, por exemplo, foram pagas 434.246 indenizações relacionadas a acidentes de trânsito no Brasil, sendo 80\% decorrentes de invalidez permanente, $13 \%$ de despesas médicas e $7 \%$ por morte (33.547). Além dos óbitos, os acidentes com vítimas não fatais correspondem a uma parcela significativa do número de ocorrências.

Segundo o relatório anual da OMS de 2015, o Brasil ocupa o 3 o lugar entre os países com o maior número absoluto de mortes causadas pelo trânsito, com taxa de mortalidade de 23,4 para cada 100 mil habitantes (WHO, 2015). No ano de 2015, segundo o Ministério da Saúde (DATASUS, 2017), foram registrados no país 38.651 óbitos decorrentes de acidentes de trânsito, dos quais 21,4\% correspondiam a vítimas mais vulneráveis do sistema de transportes (pedestres e ciclistas). Estes constituem o escopo central do presente trabalho, haja vista a importância desses modos de transporte para a melhoria da mobilidade urbana, por serem não motorizados, porém os mais vulneráveis nos deslocamentos urbanos.

No ano de 2015, o número de acidentes fatais envolvendo pedestres e ciclistas somaram 8.290 vítimas (DATASUS, 2017). Outras milhares de vítimas são internadas anualmente (31.552 pedestres e 11.671 de ciclistas no ano de 2016) (DATASUS, 2017).

Se, por um lado, há uma necessidade premente de desenvolver ações visando à redução desses acidentes, em especial os que envolvem fatalidades, é fundamental, por outro lado, que haja estudos aprofundados relacionados ao diagnóstico e ao prognóstico, além de permitir que as intervenções sejam determinadas com base em evidências (Gawryszewski et al., 2009). Carvalho (2015) aponta para o grave problema da qualidade e da disponibilidade de estatísticas de acidentes de trânsito, havendo carência de melhoria do tratamento estatístico e conhecimento de metodologias aplicáveis em subsídio à tomada de decisão pública, como valoração social e econômica decorrente desses acidentes. 
Para tal, o objetivo da pesquisa é desenvolver metodologia para estimar os custos gerados devido a acidentes de trânsito envolvendo pedestres e ciclistas. Como estudo de caso, serão utilizados dados do Estado de Santa Catarina, haja vista a necessidade de suprir lacunas acerca da dimensão desse problema e de haver estudos que sejam desagregados geograficamente (por Estados, municípios ou setores censitários) para áreas que permitam melhor identificar as carências específicas e as prioridades. O estudo utilizará dados contidos no Sistema de Informações de Saúde vinculado ao Departamento de Informática do Sistema Único de Saúde (Datasus), considerando dados de mortalidade e morbidade hospitalar a serem compilados. Para estimar os custos, a valoração de óbitos se deu pelo método do capital humano, e os valores associados à morbidade foram obtidos com base nos gastos com internações que constam no sistema do Datasus. A pesquisa foi desenvolvida visando suprir essas lacunas, tanto no sentido do entendimento de averiguar quais as taxas e os registros de acidentes de trânsito na área de estudo como para a validação de metodologia para a valoração dos custos não contabilizados (externalidades não incorporadas no planejamento urbano).

\section{Métodos de estimativa de custo de acidentes}

A monetização dos custos de acidentes, sobretudo de vítimas fatais, é complexa e criticada por alguns autores que argumentam questões de incompatibilidade ética acerca de valoração de vidas (Mishan, 1971; Hauer, 1994), em parte pela dificuldade de monetizar a dor e o sofrimento (Mohan, 2002).

Em contrapartida, diversos autores defendem a pertinência de tais abordagens. A quantificação dos acidentes de trânsito mostra-se valorosa para a avaliação estratégica das necessidades de melhorias no sistema de mobilidade urbana ou para a avaliação das consequências ou alternativas de projetos governamentais (Bardal \& Jørgensen, 2017).

Com frequência, as opções efetuadas relacionadas aos transportes têm um custo social superior ao benefício social, gerando ineficiência social, o que reduz o bem-estar coletivo. Com isso, os elevados riscos dos deslocamentos rodoviários e o custo social implícito dos acidentes não estão refletidos no produto e no rendimento nacional (Donário \& Santos, 2012).

As análises de custos permitem, dessa forma, subsidiar contramedidas com custo efetivo para a segurança rodoviária e também para justificar despesas com elas (Mohan, 2002), além de que a priorização de investimentos é por vezes subestimada por governantes (Bastida et al., 2004). Ressalta-se também a importância de discutir os problemas de internalização dos custos externos do sistema de transporte, permitindo um envolvimento mais efetivo das infraestruturas de transporte nos programas de desenvolvimento governamental (Pukalskas et al., 2015).

As abordagens para estimativa de custos são variadas. 0 valor de uma vida pode ser calculado por meio de uma metodologia ou mesmo por conjunto de métodos Elvik (2000). No geral, os custos podem ser caracterizados como diretos e indiretos. 0 primeiro diz respeito aos valores médicos e não médicos relacionados ao diagnóstico, ao tratamento, à recuperação e à reabilitação da doença. Já o segundo referese à perda de produção e de produtividade decorrente do problema de saúde (Jorge \& Koizumi, 2004).

Impactos econômicos dos acidentes de trânsito também podem ser estimados em custos diretos, custos de produção e custos psicológicos. 0 primeiro representa os danos e os reparos materiais, os custos de oportunidade de órgãos de atendimento, os custos de ações jurídicas entre envolvidos, os custos dos serviços médico-hospitalares de tratamento e/ou outros custos decorrentes de óbito. 0 segundo refere-se aos valores de perda do trabalho produtivo dos indivíduos, ao enfraquecimento físico produzido pelo acidente, às perdas de tempo provocadas pelo acidente e aos custos transitórios (recrutamento e treinamento de pessoal substituto). Já o terceiro reflete a aversão da maioria dos indivíduos aos riscos de segurança de si e dos outros (Barnet \& Clough, 1999).

Os custos diretos também são tidos por alguns autores como custos de restituição (CR), os quais consideram valores relacionados aos custos médicos, aos danos materiais, aos custos administrativos etc. (Antic et al., 2011). 
Outro método que vem sendo muito utilizado é a abordagem de disposição a pagar (DP), utilizada para estimar o valor da perda de qualidade de vida, a qual poderá considerar informações obtidas de indivíduos ou da sociedade com base no risco reduzido da avaliação implícita nas decisões públicas (AlMasaeid et al., 1999). No Brasil, destaca-se trabalho realizado em Porto Alegre por meio da metodologia de preferência declarada, obtendo-se valores estatísticos da vida entre $\mathrm{R} \$ 359$ a $\mathrm{R} \$ 998$ mil (Rosa, 2006).

Enquanto a abordagem metodológica da DP é empregada para estimar o valor da perda de qualidade de vida, o capital humano $(\mathrm{CH})$, de modo geral, é utilizado para calcular o valor da capacidade produtiva perdida devido a acidentes de trânsito, sendo esse último um dos métodos mais confiáveis e internamente consistentes (Alrukaibi et al., 2015). A abordagem do capital humano não só fornece um conjunto de números confiável como também tem um robusto fundamento teórico e, como tal, pode fornecer informações úteis aos tomadores de decisão no setor público (Robinson, 1986).

Os componentes de custo podem ser agrupados em três categorias: a categoria de custo humano (perda de produtividade, qualidade de vida e custo médico), a categoria de custo de danos materiais (danos veiculares e não veiculares) e a categoria de custo geral de acidentes de trânsito (administração de custo, custo de impacto ambiental e custo de atraso de viagem) (Alrukaibi et al., 2015).

A perda da produtividade é, geralmente, o fator mais adotado nas estimativas monetárias, além de dominar a estrutura de custos de vítimas fatais com aproximadamente $81 \%$ do valor. Para as vítimas não fatais, os principais custos são produtividade (38\%) e gastos médicos (26\%) (Blincoe et al., 2002). Outros estudos chegam a inferir que a perda de custo de produção pode representar $59 \%$ dos custos totais da mortalidade por acidentes (Al-Masaeid et al., 1999). No Brasil, segundo estudos do IPEA e da ANTP, os custos envolvendo acidentes (fatais e não fatais) se dão, principalmente, pela perda de produção (42,8\%), danos à propriedade (30\%), custos médico-hospitalares $(15,9 \%)$ e outros custos $(11,3 \%)$ (que contemplam impacto, família, atendimento policial, remoção, processos judiciais, previdenciários) (Lima, 2003; IPEA, 2015).

A perda de produtividade varia de acordo com a idade, a renda da vítima e a gravidade dos acidentes. Os principais dados necessários para a estimativa de perda de produção são os rendimentos médios das vítimas e a quantidade de tempo perdido devido a acidentes de trânsito. Para a vítima fatal, o tempo perdido é baseado nos anos perdidos que se poderia trabalhar no futuro, enquanto, para a vítima acidentada, o tempo perdido é calculado com base nos dias perdidos que se trabalharia no futuro (Alrukaibi et al., 2015).

\section{Metodologia}

No estudo, foram analisados todos os óbitos e as internações relacionados a acidentes de trânsito (causas externas), tendo por vítimas pedestres e ciclistas, descritos pelo Datasus como: pedestres traumatizados por acidente de transportes (categorias V01 a V09 do CID-1010) e ciclistas traumatizados por acidente de transportes (categorias V10 a V19 do CID-10).

A desagregação geográfica do estudo comporta análise em nível municipal pertencentes à Santa Catarina. Esse Estado possui 295 municípios, sendo registradas internações em 240 e 229 municípios e óbitos em 252 e 155 municípios, respectivamente, para acidentes com pedestres e ciclistas.

Para os registros de óbitos contidos no Datasus, foram selecionados dados por local de ocorrência, e para internações, registros por local de residência, uma vez que, selecionando-se por local de internação, poderia ocasionar um maior desvio na inferência do local de ocorrência quando comparado com o local de residência, já que grande parte dos municípios do Estado é de pequeno porte (36,9\% dos municípios tinham menos de 5 mil habitantes segundo Censo Demográfico) (IBGE, 2010) e não possui uma estrutura

I CID-10: Classificação Estatística Internacional de Doenças e Problemas Relacionados com a Saúde. 
médico-hospitalar adequada para tratamentos de lesões de gravidade severas, sendo utilizadas redes de saúde de municípios próximos.

Os custos decorrentes de acidentes envolvendo pedestres e ciclistas foram elencados com base nos valores despendidos para morbidade (despesas médico-hospitalares), enquanto os valores relacionados aos óbitos, no método do capital humano, especificamente nos custos de produtividade das vítimas (Figura 1).

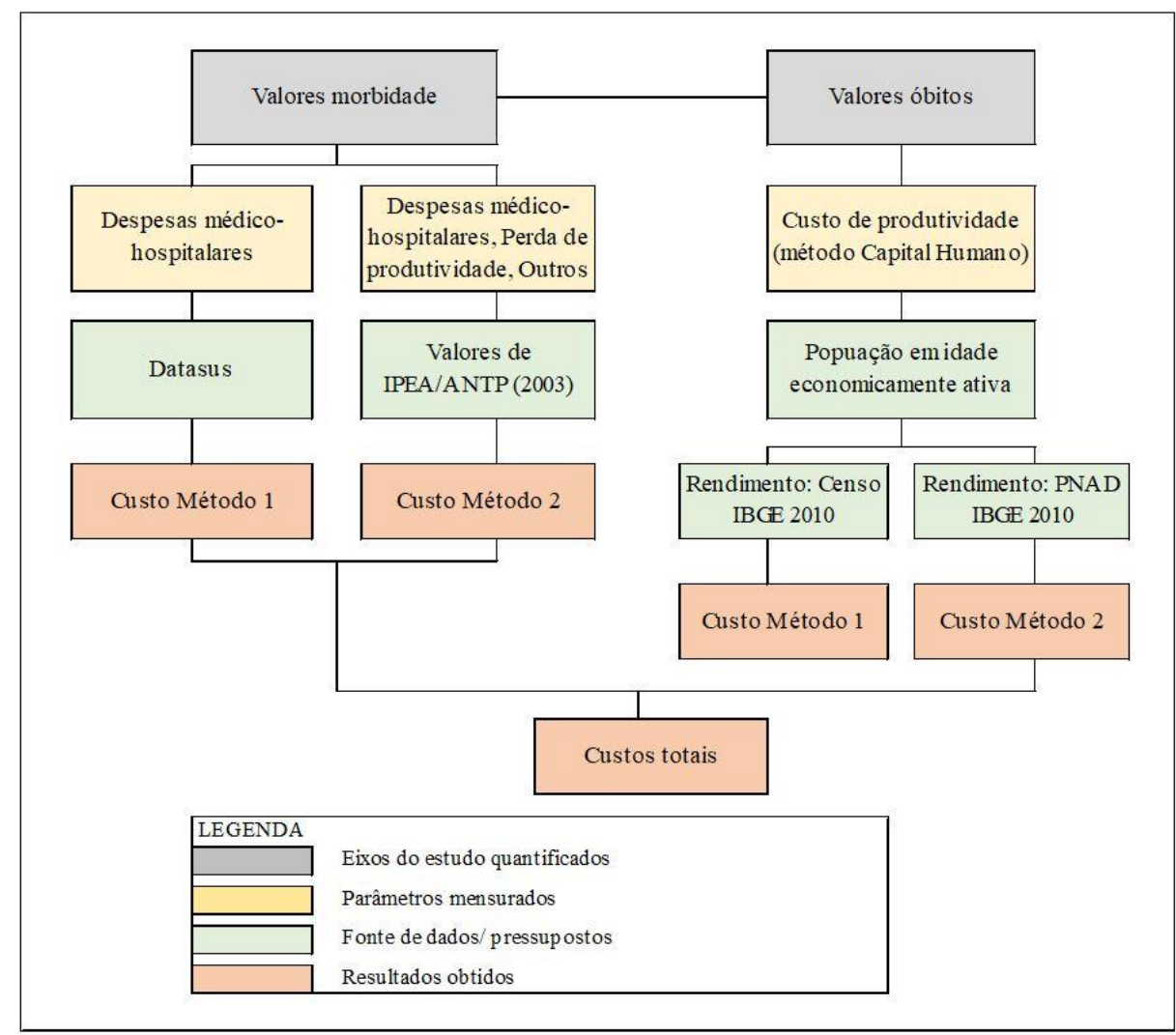

Figura 1 - Fluxograma das etapas de desenvolvimento da pesquisa. Fonte: elaborada pelo autor.

\section{Gastos decorrentes com internações}

Para os valores relacionados às vítimas não fatais (morbidade hospitalar), foram somados, além das despesas médico-hospitalares (fornecidas pelo Datasus), valores aproximados de perda de produtividade e outras despesas, com valores referenciais fornecidos pelo IPEA e pela ANTP (Lima, 2003), consistindo, dessa forma, em duas metodologias, denominadas método 1 e método 2 , respectivamente.

\section{Método 1: Despesas médico-hospitalares}

Os custos são registrados pelo Datasus, considerando despesas com serviços hospitalares, serviços profissionais, valores totais e valores médios. Para estimar valor referencial decorrente das internações, utilizou-se a média de despesas dos municípios ao longo do período de registro dos dados (1997-2015) dos acidentes com pedestres e ciclistas, obtendo-se valor unitário por vítima para o Estado de estudo.

0 valor total registrado é o custo referente à Autorização de Internação Hospitalar (AIH) aprovada no período. Segundo Jorge \& Koizumi (2004), esse documento habilita a internação do paciente e gera 
valores para pagamento. A AIH é preenchida pelo estabelecimento hospitalar prestador do serviço e enviada ao gestor municipal/estadual para o processamento e demais cumprimentos de suas finalidades.

Esse valor não obrigatoriamente corresponde ao valor repassado ao estabelecimento, pois, dependendo da situação das unidades, este recebe recursos orçamentários ou tem retenções e pagamentos de incentivos (aqui não apresentados). Portanto, esse valor deve ser considerado como o valor aprovado da produção. 0 valor total é composto pela soma do valor de serviços hospitalares (SH) e valor dos serviços profissionais (SP) (Datasus, 2017).

\section{Método 2: Despesas médico-hospitalares, perda de produtividade e outros custos}

Além da verificação dos custos médico-hospitalares registrados pelo Datasus, foram estimados outros custos para que fosse possível, além da comparação das duas metodologias, buscar uma aproximação mais fidedigna da realidade e, ao mesmo tempo, sem recorrer a metodologias complexas e inexequíveis que inviabilizariam esta análise na prática.

Para isso, foram utilizadas referências de custos indiretos fornecidos por estudos de Lima (2003) e do IPEA (2015), que classificam os custos de acidentes em quatro diferentes componentes, dos quais foram utilizados três: perda de produtividade, médico-hospitalares e outros, sendo excluídos os danos à propriedade, haja vista que a abordagem do IPEA aborda acidentes veiculares de forma geral, que é uma categoria é afetada por custos mecânicos de acidentes com veículos automotores. Como os custos médico-hospitalares são obtidos diretamente pelo Datasus, foram utilizados os valores referenciais do IPEA para as classes perda de produtividade e outros, que correspondem a 42,8 e 11,3\%, respectivamente, dos custos de acidentes sem vítimas fatais.

\section{Gastos decorrentes com óbitos}

Os custos referentes aos óbitos foram aproximados por meio dos valores em potencial de trabalho que poderiam ser produzidos pelos vitimados caso não ocorresse o acidente, tendo como pressupostos as pessoas em idade economicamente ativa e os valores de rendimento médio dessas pessoas. Para esses valores, foram encontradas duas variações nas fontes de dados de rendimentos, PNAD (IBGE, 2017) e Censo do IBGE (IBGE, 2010), sendo adotadas as duas para a comparação.

Os valores relacionados aos óbitos de acidentes são obtidos por meio de um valor estatístico da vida (VEV), que corresponde ao montante de recursos que a sociedade deseja investir para salvar a vida de uma pessoa indeterminada (Contador \& Oliveira, 2015). Para isso, utilizou-se a abordagem do capital humano, considerando como fundamento de composição de custo os valores médios de produtividade das pessoas que vieram à óbito, a idade média da pessoa no ano do óbito e o valor médio salarial da região de estudo. Segundo Carvalho (2015), essa metodologia está fundamentada no valor presente da renda que a pessoa falecida teria gerado caso o acidente não ocorresse.

Devido à complexidade dessa quantificação, a pesquisa utilizou como critério apenas a população em idade economicamente ativa para a ponderação. Foram consideradas pessoas com idade entre 15 e 69 anos (o sistema do Datasus fornece o número de pessoas por grupos de idade: 15 a 19 anos, 20 a 29 anos, 30 a 39 anos, 40 a 49 anos, 50 a 59 anos e 60 a 69 anos). Para viabilizar os cálculos, foi necessário estabelecer uma idade dentro dessa faixa etária, sendo adotada a média, uma vez que a disponibilidade de dados é por faixa etária, mas não disponibilizada a idade de cada vítima.

Com relação aos rendimentos, foram utilizadas duas fontes distintas de rendimentos: Censo Demográfico e da Pesquisa Nacional por Amostra de Domicílios Contínua (PNAD), ambas realizadas pelo IBGE, que possuem distinções metodológicas que impactam nos valores médios de rendimento. Dessa forma, os resultados foram organizados com base em dois cenários, cada um em acordo com a fonte de dados provenientes dos valores de rendimento disponibilizados pelo Censo ou pela PNAD do IBGE. 


\section{Método 1: Rendimento médio: Censo/IBGE}

Dados referentes ao Censo Demográfico do IBGE (2010) exprimem a renda média anual por domicílio; dessa forma, assumiu-se que a fonte de renda é obtida por duas pessoas por domićlílo (utilizando como referência a predominância do tipo de composição familiar em que $81,5 \%$ das famílias são compostas por casal, com ou sem filhos, segundo dados do Censo do IBGE (2010), além de considerar a densidade domiciliar média de 3,1 pessoas/domicílio no Estado).

Esses valores de rendimentos foram corrigidos ao longo do tempo com aumento de ren da de $5 \%$ ao ano, ou seja, ganhos futuros de $3 \%$ e crescimento da produtividade de $2 \%$ ao ano, segundo Bastida et al. (2004). Ainda, esses valores foram ajustados à taxa de desemprego média no período (segundo Datasus, a taxa de desemprego para o Estado de Santa Catarina no ano de 2010 foi de 3,6\%). É importante considerar a parcela da população desocupada, já que, assumindo-se o pleno emprego, a renda média seria maior, e, dessa forma, com 3,6\% pessoas a mais trabalhando, a renda real média potencial seria incrementada.

\section{Método 2: Rendimento médio: PNAD/IBGE}

A PNAD possui caráter amostral, de divulgação trimestral pelo IBGE, com valores médios por Estado descrito na seguinte categoria: Rendimento médio do trabalho principal, habitualmente recebido por mês, pelas pessoas de 14 anos ou mais de idade, ocupadas na semana de referência, com rendimento de trabalho (IBGE, 2017).

A partir da definição dos rendimentos médios mensais para cada fonte de dados, foi obtida a média anual de rendimento (considerando 12 meses). Esta foi projetada para horizonte de tempo (diferença da data final da idade economicamente ativa e da data de óbito), com uma taxa de incremento de $5 \%$ ao ano, já descrita, por meio da equação de acúmulo de capital ou denominada método da aplicação com depósitos regulares.

0 valor final corresponde à soma de uma progressão geométrica formada por $n$ pagamentos iguais a $p$ realizados no início de cada período e corrigidos até o final dos $n$ períodos (Banco Central do Brasil, 2017).

0 valor resultante é o valor médio estatístico da vida para a respectiva idade de óbito, a qual, quando multiplicada pelo número de vítimas fatais nessa faixa de idade, exprime o valor total do custo com óbitos no respectivo ano e grupo de idade. Por conseguinte, a soma dos valores por grupo de idade exprime o valor total dos custos.

\section{Resultados e discussão}

\section{Custos dos acidentes sem fatalidades}

Observou-se que o número de ciclistas acidentados é superior ao de pedestres no Estado de Santa Catarina tanto para o ano de 2015 como para o de 2016, ao contrário do número de fatalidades, em que o número de pedestres é superior ao de ciclistas. Importante destacar que, mesmo observando uma diminuição no total de acidentes envolvendo pedestres, houve um aumento nos ciclistas vitimados, podendo deduzir que isso se deva ao maior número de cliclistas presenciado nos últimos anos.

Com relação aos custos, o método 1, baseado nas despesas médico-hospitalares, mostrou um valor médio de $\mathrm{R} \$ 2.253,87$ para pedestres e $\mathrm{R} \$ 2.116,83$ para ciclistas, o que fornece custos de 1,18 milhão e 1,87 milhão, respectivamente, para pedestres e ciclistas, totalizando $\mathrm{R} \$ 3,05$ milhões para o ano de 2016, no Estado de Santa Catarina (Tabela 1). 
Tabela 1 - Custos dos acidentes com pedestres e ciclistas sem fatalidades nos dois métodos utilizados para cada tipo de acidente no Estado de Santa Catarina

\begin{tabular}{ccccc}
\hline Referência de dados & Tipo de acidente & Pedestres & Ciclistas & Total \\
\hline \multirow{3}{*}{ DATASUS } & Número de internações 20151 & 604 & 719 & 1.323 \\
& Número de internações 20161 & 550 & 865 & 1.415 \\
& Custo médio médico-hospitalar & $\mathrm{R} \$ 2.253,87$ & $\mathrm{R} \$ 2.116,83$ & - \\
& Custo médico-hospitalar 20152 & $\mathrm{R} \$ 1.460 .255,98$ & $\mathrm{R} \$ 1.344 .406,29$ & $\mathrm{R} \$ 2.804 .662,27$ \\
& Custo médico-hospitalar 20162 & $\mathrm{R} \$ 1.181 .466,91$ & $\mathrm{R} \$ 1.872 .739,30$ & $\mathrm{R} \$ 3.054 .206,21$ \\
\hline \multirow{2}{*}{ IPEA/ANTP } & Custo total acidente sem fatalidade & $\mathrm{R} \$ 9.922,71$ & $\mathrm{R} \$ 9.319,36$ & - \\
& Custo total estimado 2015 & $\mathrm{R} \$ 5.993 .315,89$ & $\mathrm{R} \$ 6.700 .618,78$ & $\mathrm{R} \$ 12.693 .934,67$ \\
& Custo total estimado 2016 & $\mathrm{R} \$ 5.457 .489,63$ & $\mathrm{R} \$ 8.061 .245,12$ & $\mathrm{R} \$ 13.518 .734,76$ \\
\hline
\end{tabular}

'Dados Datasus/MS. ${ }^{2}$ Valores reais obtidos pelo sistema DATASUS e não resultantes do produto do número de vítimas pelo valor médio do custo do tratamento. Fonte: elaborada pelo autor.

Com base no método 2 (IPEA e ANTP), que estima custos excedentes aos médico-hospitalares, foram obtidos os valores $\mathrm{R} \$ 9.922,71$ para pedestres e $\mathrm{R} \$ 9.319,36$ para ciclistas, o que fornece custos de 5,45 milhões e 6,70 milhões, respectivamente, para pedestres e ciclistas, totalizando $\mathrm{R} \$ 13,52$ milhões para o ano de 2016.

Apresentaram-se os dados de 2015 para poder verificar a contabilidade total de gastos (acidentes com vítimas e óbitos), haja vista que dados de internações estão disponíveis até o ano de 2016, e de óbitos, até 2015.

\section{Custos dos óbitos}

Para o método 1, adotou-se o rendimento nominal fornecido pelo Censo Demográfico. Os valores são parametrizados por grupos de idade, variando entre $\mathrm{R} \$ 1.463,15$ a $\mathrm{R} \$ 1.934,57$ mensais per capita. Esses valores ponderados pelos anos produtivos futuros de cada vítima, por grupo de idade, gerou valores de $\mathrm{R} \$ 120.193,88$ a $\mathrm{R} \$ 1.227 .537,30$ da maior para a menor idade, respectivamente (vide Tabela 2). A soma resultou em $\mathrm{R} \$ 110,01$ milhões decorrentes de óbitos de pedestres e $\mathrm{R} \$ 44,98$ milhões para ciclistas, totalizando $\mathrm{R}$ \$ 154,99 milhões, 7,4\% menor em relação à utilização de valores de rendimento da PNAD.

Tabela 2 - Custos de óbitos de acidentes com pedestres e ciclistas nos dois métodos utilizados para cada tipo de acidente no Estado de Santa Catarina

\begin{tabular}{|c|c|c|c|c|c|c|}
\hline \multicolumn{7}{|c|}{ Método: Custos com base no Censo (IBGE, 2010) por grupo de idade } \\
\hline Faixa etária do óbito & 15 a 19 anos & 20 a 29 anos & 30 a 39 anos & 40 a 49 anos & 50 a 59 anos & 60 a 69 anos \\
\hline Óbito de pedestres' & 7 & 25 & 25 & 45 & 45 & 32 \\
\hline Média mensal & $R \$ 1.463,15$ & $R \$ 1.805,32$ & $R \$ 1.671,14$ & $R \$ 1.750,39$ & $R \$ 1.934,57$ & $R \$ 1.770,78$ \\
\hline $\begin{array}{l}\text { Média anual } 2015 \\
\text { Valor médio de }\end{array}$ & $R \$ 17.557,83$ & $R \$ 21.663,82$ & $R \$ 20.053,74$ & $\mathrm{R} \$ 21.004,71$ & $R \$ 23.214,80$ & $\mathrm{R} \$ 21.249,37$ \\
\hline $\begin{array}{l}\text { óbito por pessoa e } \\
\text { faixa etária }\end{array}$ & $\mathrm{R} \$ 1.227 .537,30$ & $\mathrm{R} \$ 1.248 .604,13$ & $R \$ 854.940,55$ & $R \$ 610.326,56$ & $R \$ 389.363,82$ & $R \$ 120.193,88$ \\
\hline $\begin{array}{l}\text { Valor médio de } \\
\text { óbito por faixa etária } \\
\text { Gasto total no }\end{array}$ & $\mathrm{R} \$ 8.592 .761,08$ & $R \$ 31.215 .103,28$ & $\mathrm{R} \$ 21.373 .513,70$ & $R \$ 27.464 .695,10$ & $\mathrm{R} \$ 17.521 .372,03$ & $R \$ 3.846 .204,27$ \\
\hline $\begin{array}{l}\text { Estado em } 2015 \\
\text { para pedestres }\end{array}$ & \multicolumn{6}{|r|}{$\mathrm{R} \$ 110.013 .649,47$} \\
\hline Faixa etária do óbito & 15 a 19 anos & 20 a 29 anos & 30 a 39 anos & 40 a 49 anos & 50 a 59 anos & 60 a 69 anos \\
\hline Óbitos de ciclistas' & 10 & 7 & 9 & 8 & 24 & 17 \\
\hline Média mensal & $R \$ 1.463,15$ & $R \$ 1.805,32$ & $R \$ 1.671,14$ & $R \$ 1.750,39$ & $R \$ 1.934,57$ & $R \$ 1.770,78$ \\
\hline $\begin{array}{l}\text { Média anual } 2015 \\
\text { Valor médio de }\end{array}$ & $R \$ 17.557,83$ & $R \$ 21.663,82$ & $R \$ 20.053,74$ & $R \$ 21.004,71$ & $R \$ 23.214,80$ & $R \$ 21.249,37$ \\
\hline $\begin{array}{l}\text { óbito por pessoa e } \\
\text { faixa etária }\end{array}$ & $R \$ 1.227 .537,30$ & $R \$ 1.248 .604,13$ & $R \$ 854.940,55$ & $R \$ 610.326,56$ & $R \$ 389.363,82$ & $R \$ 120.193,88$ \\
\hline $\begin{array}{l}\text { Valor médio de } \\
\text { óbito por faixa etária } \\
\text { Gasto total no }\end{array}$ & $R \$ 12.275 .372,98$ & $\mathrm{R} \$ 8.740 .228,92$ & $\mathrm{R} \$ 7.694 .464,93$ & $R \$ 4.882 .612,46$ & $\mathrm{R} \$ 9.344 .731,75$ & $R \$ 2.043 .296,02$ \\
\hline $\begin{array}{l}\text { Estado em } 2015 \\
\text { para ciclistas }\end{array}$ & & & & & & $\mathrm{R} \$ 44.980 .707,06$ \\
\hline
\end{tabular}


Tabela 2 - Continuação...

\begin{tabular}{|c|c|c|c|c|c|c|}
\hline \multicolumn{7}{|c|}{ Méłodo: Média anual nominal em SC, PNAD/IBGE } \\
\hline Faixa etária do óbito & 15 a 19 anos & 20 a 29 anos & 30 a 39 anos & 40 a 49 anos & 50 a 59 anos & 60 a 69 anos \\
\hline Óbito de pedestres 1 & 7 & 25 & 25 & 45 & 45 & 32 \\
\hline Média mensal & $\mathrm{R} \$ 1.871,50$ & $\mathrm{R} \$ 1.871,50$ & $\mathrm{R} \$ 1.871,50$ & $\mathrm{R} \$ 1.871,50$ & $\mathrm{R} \$ 1.871,50$ & $\mathrm{R} \$ 1.871,50$ \\
\hline $\begin{array}{l}\text { Média anual } 2015 \\
\text { Valor médio de }\end{array}$ & $R \$ 22.458,00$ & $R \$ 22.458,00$ & $R \$ 22.458,00$ & $R \$ 22.458,00$ & $\mathrm{R} \$ 22.458,00$ & $R \$ 22.458,00$ \\
\hline $\begin{array}{c}\text { óbito por pessoa e } \\
\text { faixa etária }\end{array}$ & $R \$ 1.570 .127,51$ & $\mathrm{R} \$ 1.294 .377,29$ & $R \$ 957.440,25$ & $R \$ 652.554,31$ & $R \$ 376.670,62$ & $R \$ 127.030,34$ \\
\hline $\begin{array}{l}\text { Valor médio de } \\
\text { óbito por faixa etária } \\
\text { Gasto total no }\end{array}$ & $R \$ 10.990 .892,56$ & $R \$ 32.359 .432,20$ & $R \$ 23.936 .006,23$ & $R \$ 29.364 .944,08$ & $R \$ 16.950 .177,94$ & $R \$ 4.064 .970,80$ \\
\hline $\begin{array}{l}\text { Estado em } 2015 \\
\text { para pedestres }\end{array}$ & & & & & & $2 \$ 117.666 .423,80$ \\
\hline Faixa etária do óbito & 15 a 19 anos & 20 a 29 anos & 30 a 39 anos & 40 a 49 anos & 50 a 59 anos & 60 a 69 anos \\
\hline Óbitos de ciclistas 1 & 10 & 7 & 9 & 8 & 24 & 17 \\
\hline Média mensal & $\mathrm{R} \$ 1.871,50$ & $\mathrm{R} \$ 1.871,50$ & $\mathrm{R} \$ 1.871,50$ & $\mathrm{R} \$ 1.871,50$ & $\mathrm{R} \$ 1.871,50$ & $\mathrm{R} \$ 1.871,50$ \\
\hline $\begin{array}{l}\text { Média anual } 2015 \\
\text { Valor médio de }\end{array}$ & $\mathrm{R} \$ 22.458,00$ & $R \$ 22.458,00$ & $\mathrm{R} \$ 22.458,00$ & $\mathrm{R} \$ 22.458,00$ & $\mathrm{R} \$ 22.458,00$ & $R \$ 22.458,00$ \\
\hline $\begin{array}{l}\text { óbito por pessoa e } \\
\text { faixa etária }\end{array}$ & $R \$ 1.570 .127,51$ & $\mathrm{R} \$ 1.294 .377,29$ & $R \$ 957.440,25$ & $R \$ 652.554,31$ & $R \$ 376.670,62$ & $R \$ 127.030,34$ \\
\hline $\begin{array}{l}\text { Valor médio de } \\
\text { óbito por faixa etária } \\
\text { Gasto total no }\end{array}$ & $R \$ 15.701 .275,08$ & $R \$ 9.060 .641,02$ & $\mathrm{R} \$ 8.616 .962,24$ & $R \$ 5.220 .434,50$ & $R \$ 9.040 .094,90$ & $R \$ 2.159 .515,74$ \\
\hline $\begin{array}{l}\text { Estado em } 2015 \\
\text { para ciclistas }\end{array}$ & & & & & & $\$ 49.798 .923,48$ \\
\hline
\end{tabular}

IDados Datasus/MS. Fonte: elaborada pelo autor.

Para essa estimativa utilizando o método 2, considerou-se o rendimento médio mensal do trabalho principal fornecido pela pesquisa PNAD (IBGE, 2017) de $\mathrm{R} \$ 1.871,50$ e anual de $\mathrm{R} \$ 22.458,00$ (Tabela 2).

Esses valores ponderados pela média de anos produtivos e pelo número de vítimas resultaram em custos que variaram de $\mathrm{R} \$ 127.030,34$ a $\mathrm{R} \$ 1.570 .127,51$ por vítima, dependendo da maior ou menor idade no momento da ocorrência, respectivamente. A soma resultou em um valor de R \$117,7 milhões para óbitos de pedestres e $\mathrm{R} \$ 49,8$ milhões para ciclistas, totalizando $\mathrm{R} \$ 167,5$ milhões, considerando todo o Estado de Santa Catarina.

\section{Custos totais}

A compilação dos custos resultou em cenários por causa das diferentes possibilidades para as estimativas. Isso gerou uma variação entre $\mathrm{R}$ \$ 157,8 e R \$ 180,2 milhões, apresentado uma diferença de aproximadamente 12,4\% (Tabela 3). Com relação às vítimas não fatais (acidentes com lesão), a estimativa com dados de despesas médico-hospitalares (Datasus) forneceu um valor inferior ao método do IPEA e da ANTP, em razão de esse último incorporar despesas indiretas. Os valores estimados de acidentes com vítimas fatais (óbitos) correspondem aos maiores custos por incorporar um VEV. 0 método que utilizou dados de rendimentos com base na PNAD do IBGE gerou maiores valores do que o que utiliza dados censitários (diferença de 8,04\%).

Tabela 3 - Custos totais, considerando os dois métodos utilizados em cada tipo de acidente para o Estado de Santa Catarina no ano de 2015

\begin{tabular}{|c|c|c|c|c|}
\hline \multicolumn{5}{|c|}{ Custos totais no ano de 2015 para o Estado de Santa Catarina (R\$) } \\
\hline Tipo de acidente & Método & Pedestre & Ciclista & Total \\
\hline \multirow{2}{*}{ Lesão } & DATASUS & $\mathrm{R} \$ 1.460 .255,98$ & $\mathrm{R} \$ 1.344 .406,29$ & $\mathrm{R} \$ 2.804 .662,27$ \\
\hline & IPEA/ANTP & $R \$ 5.993 .315,89$ & $\mathrm{R} \$ 6.700 .618,78$ & $R \$ 12.693 .934,67$ \\
\hline \multirow{4}{*}{ Óbito } & Censo Demográfico/IBGE & $R \$ 110.013 .649,47$ & $\mathrm{R} \$ 44.980 .707,06$ & $R \$ 154.994 .356,52$ \\
\hline & PNAD/IBGE & $R \$ 117.666 .423,80$ & $R \$ 49.798 .923,48$ & $R \$ 167.465 .347,28$ \\
\hline & Total mínimo & $\mathrm{R} \$ 111.473 .905,45$ & $R \$ 46.325 .113,35$ & $R \$ 157.799 .018,79$ \\
\hline & Total máximo & $R \$ 123.659 .739,69$ & $R \$ 56.499 .542,26$ & $R \$ 180.159 .281,95$ \\
\hline
\end{tabular}

Fonte: elaborada pelo autor. 
Aqui, cabe uma reflexão acerca do aumento do número de vítimas ao longo dos anos. Considerando os casos registrados nos últimos dez anos, por exemplo, haveria um custo aproximado de $\mathrm{R} \$ 2.519,5$ milhões e de $\mathrm{R}$ \$29,4 milhões, respectivamente, para óbitos e internações (custos médico-hospitalares). Já para os valores com base nos custos diretos e indiretos (método 2), os custos estimados atingiram $\mathrm{R} \$ 2.722,3$ milhões e $\mathrm{R} \$ 137,1$ milhões, respectivamente, para custos de acidentes com óbitos e sem fatalidades. Isso aponta para uma faixa de valores entre $\mathrm{R} \$ 2,55$ e $\mathrm{R} \$ 2,86$ bilhões no período apenas considerando vítimas ciclistas e pedestres, o que representa aproximadamente de 1,2\% do atual PIB do Estado (correspondente a R $\$ 242,5$ bilhões em 2014 (IBGE, 2017).

Porém, ao comparar esses valores com os gastos públicos do Estado, entende-se melhor a proporção desses valores. Segundo o Portal Transparência/SC (SIGEF, 2016), no ano de 2016, o Estado empenhou um total de $\mathrm{R} \$ 20,1$ bilhões de recursos, dos quais $\mathrm{R} \$ 2,85$ bilhões referentes à saúde. Dessa forma, os custos estimados dos acidentes no mesmo ano corresponderam a 14\% do total disponível pelo Estado e a quase a totalidade dos recursos gastos com a saúde pública, quando comparado apenas com os gastos com saúde pública.

Esses valores estimados podem servir de base para tomadores de decisão, visando à melhoria das ações relacionadas à segurança viária, e de subsídios à análise de medidas estruturais a serem implementadas. As estimativas realizadas mostraram valores semelhantes entre si, porém que podem ser aprimorados de acordo com dados específicos de cada região, onde valores de lesões podem variar de $\mathrm{R} \$ 2$ mil (considerando apenas despesas médico-hospitalares) a quase $\mathrm{R} \$ 10$ mil por vítima (Figura 2 ).

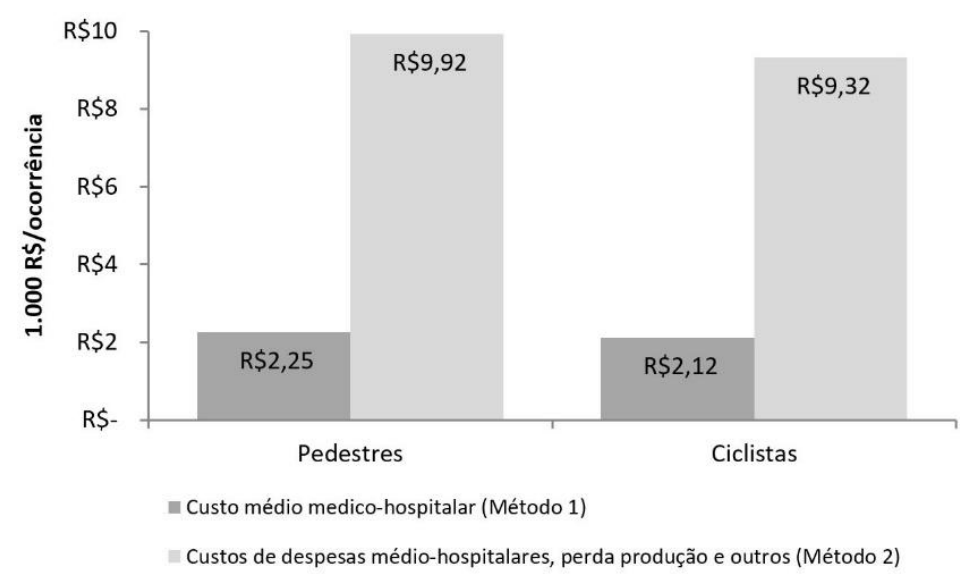

Figura 2 - Custos médios dos valores estimados por vítima para lesões resultantes de acidentes envolvendo pedestres e ciclistas. Fonte: elaborada pelo autor.

Para óbitos, os cálculos apontaram valores superiores a $\mathrm{R} \$ 20$ mil anual (por ano de vida perdido), sendo estimado cerca de $\mathrm{R} \$ 20,8$ mil para o método que utiliza dados do Censo e $\mathrm{R} \$ 22,5$ mil para o método que utiliza dados da PNAD (Figura 3). 


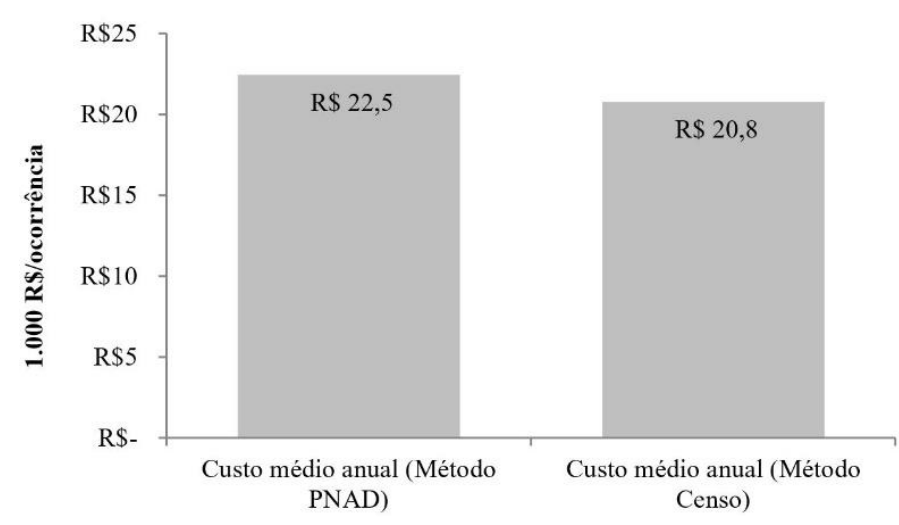

Figura 3 - Custos médios dos valores estimados por vítima para óbitos resultantes de acidentes envolvendo pedestres e ciclistas por ano de vida perdido. Fonte: elaborada pelo autor.

A metodologia desenvolvida para as estimativas do VEV mostrou-se compatível com as valorações em nível internacional. Segundo Dekker et al. (2011), esses valores, geralmente, encontram-se dentro da variação internacional dos valores atribuídos ao VEV, apesar de situarem-se abaixo da média, mas próximo aos valores atribuídos por países como Malásia (U\$ 730 mil), Reino Unido (U\$ 767 mil) ou Suíça (U\$ 971 mil). Destaca-se que, para a metodologia desenvolvida para o Brasil, os VEV variam em função da idade do óbito, apresentando uma faixa entre US\$70,4-772,1 mil para o método 1 e US\$74,7-923,6 mil para o método 2 (Tabela 4).

Tabela 4 - Exemplos de VEV em diversos estudos comparados com os valores obtidos para a pesquisa realizada

\begin{tabular}{|c|c|c|c|}
\hline \multirow{2}{*}{ País } & \multirow{2}{*}{ Ano dos dados do estudo } & \multirow{2}{*}{$\begin{array}{c}\text { Estimativa mais baixa } \\
(\text { US } \$ \times 1000)\end{array}$} & \multirow{2}{*}{$\frac{\text { Estimativa mais alta }}{(\text { US } \$ \times 1000)}$} \\
\hline & & & \\
\hline China & 1999 & 130 & 448 \\
\hline Japão & 1999 & 705 & 2.425 \\
\hline Reino Unido & 1995 & 767 & 13.110 \\
\hline Malásia & 2003 & 731 & 1.682 \\
\hline Suíça & 1994 & 971 & 1.167 \\
\hline Suécia & 2005 & 1.312 & 1.446 \\
\hline USA e Canadá & 2000 & 1.015 & 5.214 \\
\hline Rraci** & 0 & Método 1: 70,7 & Método 1: 722,1 \\
\hline Brasil** $^{*}$ & 2010 & Método 2: 74,7 & Método 2: 923,6 \\
\hline
\end{tabular}

*Valor calculado pela pesquisa ponderado por idade do óbito. Dólar de referência em 01/12/2010 = R\$ 1,70. Fonte: Dekker et al. (2011).

\section{Conclusão}

A pesquisa atingiu o objetivo de determinar uma metodologia capaz de estimar os custos decorrentes de acidentes de trânsito, podendo servir de subsídio ao planejamento urbano e à definição de políticas públicas. Mesmo com as complexidades envolvidas na estimativa, os valores gerados são passíveis de internalização em análises estratégicas de planejamento urbano e em estudos de tráfego. Os métodos apresentam diferenças de valores finais e, portanto, devem ser utilizados desde que conhecidas as suas limitações, sendo recomendada a sua difusão da abordagem em favor da compreensão por parte da sociedade e dos governos dos custos sociais decorrentes de políticas ineficientes de mobilidade urbana.

Os valores expressivos dos custos estimados pela pesquisa servem de alerta para toda a sociedade por causa do ônus causado pelos acidentes de trânsito. Essas análises podem ser utilizadas em subsídio ao planejamento de cidades, levando-se em conta dados históricos e projeções futuras. A redução de ocorrências de acidentes de pedestres e de ciclistas deverá sempre ser considerada no sentido de promover práticas exequíveis que estimulam o transporte ativo, tornando os espaços urbanos mais seguros para o deslocamento de pedestres e de ciclistas, principalmente crianças e idosos. 
Nesse sentido, a pesquisa gerou ainda novas perguntas que podem ser recomendadas para trabalhos futuros, como o aprimoramento do método, sobretudo no dimensionamento dos custos indiretos, subjetivos e psicológicos. Ainda, recomenda-se que pesquisas semelhantes sejam desenvolvidas considerando dados de transporte, relacionando a eficiência de políticas públicas e a promoção do uso de transportes ativos com a evolução de ocorrências de acidentes de trânsito.

\section{Agradecimentos}

À Coordenação de Aperfeiçoamento de Pessoal de Nível Superior (CAPES), pelo financiamento.

\section{Referências}

Al-Masaeid, H. R., Al-Mashakbeh, A. A., \& Qudah, A. M. (1999). Economic costs of traffic accidents in Jordan. Accident; Analysis and Prevention, 31(4), 347-357. http://dx.doi.org/10.1016/S0001-4575(98)00068-2. PMid:10384228.

Alrukaibi, F., Alotaibi, N., \& Almutairi, M. (2015). Methodology for calculation the traffic accidents costs. In Proceedings of 10th ISERD International Conference (pp. 46-51). Dubai: UAE.

Andrade, S. S. C. A., Jorge, M. H. P. M., Andrade, S. S. C. A., \& Jorge, M. H. P. M. (2017). Internações hospitalares por lesões decorrentes de acidente de transporte terrestre no Brasil, 2013: permanência e gastos. Epidemiologia e Serviços de Saúde : Revista do Sistema Unico de Saúde do Brasil, 26(1), 31-38. http://dx.doi.org/10.5123/S167949742017000100004. PMid:28226006.

Antic, B., Vujanic, M., Lipovac, K., \& Pesic, D. (2011). Estimation of the traffic accidents costs in Serbia by using dominant costs model. Transport, 26(4), 433-440. http://dx.doi.org/10.3846/16484142.2011.635425.

Bacchieri, G., \& Barros, A. J. D. (2011). Acidentes de trânsito no Brasil de 1998 a 2010: muitas mudanças e poucos resultados. Revista de Saude Publica, 45(5), 949-963. http://dx.doi.org/10.1590/S0034-89102011005000069. PMid:21953026.

Banco Central do Brasil. (2017). Metodologia da aplicação com depósitos regulares. Recuperado em 18 de setembro de 2017, de http://www.bcb.gov.br/pec/calculo/calc_poupanca/metodologia.asp

Bardal, K. G., \& Jørgensen, F. (2017). Valuing the risk and social costs of road traffic accidents - seasonal variation and the significance of delay costs. Transport Policy, 57, 10-19. http://dx.doi.org/10.1016/j.tranpol.2017.03.015.

Barnet, J., \& Clough, P. (1999). The full social cost of road accidents. In Road Safety Research, Policing and Education Conference (pp. 1-11). Canberra: NZ Institute of Economic Research.

Bastida, J. L., Aguilar, P. S., \& González, B. D. (2004). The economic costs of traffic accidents in Spain. The Journal of Trauma Injury Infection and Critical Care, 56(4), 883-889.

Bazani, A. (2016). Investir em transporte público é responsabilidade fiscal: 80\% dos gastos com deslocamentos vão para o transporte individual, causando prejuízos. São Paulo: Associação Nacional de Transportes Públicos.

Biffe, C. R. F., Harada, A., Bacco, A. B., Coelho, C. S., Baccarelli, J. L. F., Silva, K. L., Braccialli, L. A. D., Beloni, M., Bernardes, M. L. G., Lacerda, S. R., \& Silva, T. I. (2017). Perfil epidemiológico dos acidentes de trânsito em Marília, São Paulo, 2012. Revista Epidemiologia e Serviços de Saúde, 26(2), 389-398. http://dx.doi.org/10.5123/S167949742017000200016.

Blincoe, L., Seay, A., Zaloshnja, E., Miller, T., Romano, E., Luchter, S., \& Spicer, R. (2002). The economic impact of motor vehicle crashes 2000 (NHTSA Technical Report DOT HS 809 446). Washington: U.S. Department of Transportation.

Carvalho, L. J. (2015). Metodologia para quantificação da perda do produto com os acidentes de trânsito e evidências preliminares. Rio de Janeiro: Funenseg. 
Contador, C., \& Oliveira, N. (2015). Estatísticas da dor e da perda do futuro: novas estimativas. Rio de Janeiro: Centro de Pesquisa e Economia do Seguro - Funenseg.

Dekker, T., Brouwer, R., Hofkes, M., \& Moeltner, K. (2011). The effect of risk context on the value of a statistical life: a bayesian meta-model. Environmental and Resource Economics, 49(4), 597-624. http://dx.doi.org/10.1007/s10640011-9456-z.

Departamento de Informática do SUS - DATASUS. (2017). Morbidade hospitalar do SUS por causas externas por local de residência - a partir de 2008: notas técnicas. Rio de Janeiro: Ministério da Saúde.

Donário, A., \& Santos, R. (2012). Custo económico e social dos acidentes de viação em Portugal. Lisboa: Ediual.

Elvik, R. (2000). How much do road accidents cost the national economy? Accident; Analysis and Prevention, 32(6), 849-851. http://dx.doi.org/10.1016/S0001-4575(00)00015-4. PMid:10994613.

Garcia, L. P., Freitas, L. R. S., \& Duarte, E. C. (2013). Deaths of bicycle riders in Brazil: characteristics and trends during the period of 2000 - 2010. Revista Brasileira de Epidemiologia, 16(4), 918-929.

http://dx.doi.org/10.1590/S1415-790X2013000400012. PMid:24896597.

Gawryszewski, V. P., Coelho, H. M. M., Scarpelini, S., Zan, R., Jorge, M. H. P. M., \& Rodrigues, E. M. S. (2009). Perfil dos atendimentos a acidentes de transporte terrestre por serviços de emergência em São Paulo, 2005. Revista de Saude Publica, 43(2), 275-282. http://dx.doi.org/10.1590/S0034-89102009000200008. PMid:19287873.

Hauer, E. (1994). Can one estimate the value of life or is it better to be dead than stuck in traffic? Transportation Research, 28A(2), 109-118.

Instituto Brasileiro de Geografia e Estatística - IBGE. (2010). Tipo de composição familiar - censo demográfico 2010. Rio de Janeiro: Sistema IBGE de Recuperação Automática - SIDRA.

Instituto Brasileiro de Geografia e Estatística - IBGE. (2017). Pesquisa nacional por amostra de domicílios contínua PNAD Contínua (Divulgação Trimestral Abr-Jun). Rio de Janeito: IBGE.

Instituto de Pesquisa Econômica Aplicada - IPEA. (2015). Estimativa dos custos dos acidentes de trânsito no Brasil com base na atualização simplificada das pesquisas anteriores do ipea. Brasília: IPEA.

Jorge, M. H. P. M., \& Koizumi, M. S. (2004). Gastos governamentais do SUS com internações hospitalares por causas externas: análise no Estado de São Paulo, 2000. Revista Brasileira de Epidemiologia, 7(2), 228-238.

http://dx.doi.org/10.1590/S1415-790X2004000200012.

Lima, R. R. A. (2003) Impactos sociais e econômicos dos acidentes de trânsito nas aglomerações urbanas brasileiras: relatório executivo. Brasília: IPEA.

Litman, T. (2008). Valuing transit service quality improvements. Journal of Public Transportation, 11(2), 43-63. http://dx.doi.org/10.5038/2375-0901.11.2.3.

Litman, T. (2014). A new transit safety narrative. Journal of Public Transportation, 17(4), 114-135. http://dx.doi.org/10.5038/2375-0901.17.4.7.

Mishan, E. J. (1971). Evaluation of life and limb: a theoretical approach. Journal of Political Economy, 79(4), 687-705. http://dx.doi.org/10.1086/259784.

Mohan, D. (2002). Social Cost of Road Traffic Crashes in India. In Proceedings First Safe Community Conference on Cost of Injury (pp. 33-38). Viborg: Safe Community Conference on Cost of Injury.

Newman, P., \& Kenworthy, J. (2013). Sustainability and cities: overcoming automobile dependence (2nd ed.). Washington: Island Press.

Organisation for Economic Co-operation and Development - OECD. (2015). Road Safety Annual Report 2015. Paris: OECD.

Pukalskas, S., Peceliunas, R., Sadauskas, V., Kilikeviciene, K., \& Bogdevicius, M. (2015). The methodology for calculation of road accident costs. Transport, 30(1), 33-42. http://dx.doi.org/10.3846/16484142.2015.1020871. 
Robinson, J. C. (1986). Phiosophical origins of economic evaluation of life. The Milbank Quarterly, 64(1), 133-155. http://dx.doi.org/10.2307/3350008. PMid:3084931.

Rosa, C. N. (2006). Custo da perda de uma vida e médico-hospitalares nos acidentes de trânsito (Tese de Doutorado) Universidade Federal do Rio Grande do Sul, Porto Alegre.

Seguradora Lider. (2016). Seguro de Danos Pessoais Causados por Veículos Automotores de Via Terrestre - DPVAT: boletim estatístico (vol. 4, jan-dez). Rio de Janeiro: Lider.

Sistema Integrado de Planejamento e Gestão Fiscal - SIGEF. (2016). Portal da Transparência do Poder Executivo de Santa Catarina. Recuperado em 1 de fevereiro de 2018, de http://www.transparencia.sc.gov.br/despesa

Tischer, V. (2017). Validação de sistema de parâmetros técnicos de mobilidade urbana aplicados para sistema cicloviário. Urbe. Revista Brasileira de Gestão Urbana, 9(3), 587-604. http://dx.doi.org/10.1590/21753369.009.003.ao15.

World Health Organization - WHO. (2015). Global status report on road safety 2015. Geneva: WHO.

Editor: Fábio Duarte

Recebido: Abr. 18, 2018

Aprovado: Jul, 19, 2018 